\title{
El impuesto predial en Ciudad Juárez desde una perspectiva de equidad
}

\section{Property tax in Ciudad Juarez from an equity perspective}

\author{
Sergio Peña-Medina*
}

\begin{abstract}
Equity in the property tax system is a relevant topic that is discussed in this article. There is a difference between vertical and horizontal equity. Vertical equity points toward aspects of progressive taxation; that is, properties with a higher value should pay a higher rate than lower valued properties. Horizontal equity points towards a valuation issue; meaning that properties that are comparable should pay the same tax rate. The objective of the article is to test the hypothesis of a regressive property tax system. I argue that a regressive property tax system is unfair because higher valued properties pay a lower rate than less valued properties. The results in the research undertaken in Ciudad Juarez support the hypothesis of a regressive property tax system; thus it is important to reflect in regards to the cadastral system structure.
\end{abstract}

Keywords: property tax, regressive tax, equity.

\section{Resumen}

El aspecto de equidad en el impuesto predial es un tema relevante que se aborda en este artículo. Ésta se diferencia entre vertical y horizontal; la primera se refiere a un enfoque progresivo en donde aquellas propiedades que valen más pagan una tasa superior a las de menor valor; la equidad horizontal apunta a un tema de valuación y, en ella, las propiedades comparables pagan la misma tasa. El artículo tiene como objetivo poner a prueba la hipótesis de regresividad del impuesto predial; se argumenta que ésta es inequitativa dado que en términos proporcionales las propiedades de menor valor pagan una tasa superior a las de mayor valor. Los resultados en esta investigación soportan la hipótesis de que existe un efecto de regresividad en el impuesto predial en Ciudad Juárez; consecuentemente es importante reflexionar sobre la estructura actual del sistema catastral.

Palabras claves: impuesto predial, regresividad, equidad fiscal.

* El Colegio de la Frontera Norte, México. Correo-e: spena@colef.mx 


\section{Introducción}

Uno de los principales problemas del desarrollo en México es la debilidad estructural de las finanzas públicas municipales. En México, los municipios dependen en gran medida de transferencias federales otorgadas a través de acuerdos de coordinación fiscal; es a partir de ellas que se financia en parte el gasto operativo (ramo 28) por medio del Fondo de Aportaciones para el Fortalecimiento de los Municipios (Fortamun), el Fondo de Fortalecimiento Municipal y el gasto de inversión en infraestructura de capital (carreteras, equipamiento, etc.) y gasto social (ramo 33), entre otros. La capacidad técnica, o el costo político asociado, para aumentar la capacidad recaudatoria es una de las debilidades estructurales más serias (Colmenares-Páramo, 2005).

El impuesto predial es una de las fuentes de recursos más importantes que los municipios en México, lo mismo que en otras partes del mundo, tienen para allegarse recursos económicos y aumentar su capacidad de gestión y acción. Sin embargo, es importante enfatizar que la capacidad de gestión y acción que permite el impuesto predial conlleva, necesariamente, el planteamiento no sólo de aspectos de eficiencia y eficacia recaudatoria en la política fiscal local, sino también aspectos de equidad (Holcombe, 1983; Slack, 2013).

El objetivo del presente artículo es analizar desde una perspectiva de equidad la actual estructura del impuesto predial en Ciudad Juárez, Chihuahua. La pregunta central es ¿existe un efecto de regresividad en la estructura del impuesto predial en Ciudad Juárez? El efecto regresivo es relevante porque es una evidencia factual de inequidad, ya que en términos relativos, los dueños cuyo valor del predio es más alto son gravados a una tasa menor o igual comparada con los predios de menor valor. De manera formal la hipótesis a poner a prueba es la siguiente:

$\mathrm{H}_{1}: \mathrm{I}_{\mathrm{H}} / \mathrm{V}_{\mathrm{H}}>\mathrm{I}_{\mathrm{L}} / \mathrm{V}_{\mathrm{L}}$ (no regresividad)

Ha: $\mathrm{I}_{\mathrm{H}} / \mathrm{V}_{\mathrm{H}} \leq \mathrm{I}_{\mathrm{L}} / \mathrm{V}_{\mathrm{L}}$ (regresividad)

Donde:

$\mathrm{I}=$ impuesto total pagado

$\mathrm{V}=$ Valor del predio

$\mathrm{H}=$ predio con un valor alto

$\mathrm{L}=$ predio con un valor bajo

$\mathrm{T}=\operatorname{tas} \mathrm{a}=\mathrm{I} / \mathrm{V}$

El artículo se estructura en siete secciones: la primera es de índole contextual, en ella se abordan aspectos relacionados con la coordinación fiscal en México y la capacidad de gestión y acción de las finanzas públicas 
municipales; la segunda sección se enfoca en discutir el marco teórico de la política fiscal del impuesto predial poniendo especial énfasis en el aspecto de equidad. La tercera sección se dedica a aspectos econométricos que se refieren a la medición del concepto de equidad en el impuesto predial; la siguiente sección desarrolla la descripción de los datos y las metodologías de cuadrados mínimos ordinarios que se emplearon para medir la equidad del impuesto predial en Ciudad Juárez. Los datos y aspectos metodológicos son abordados de manera amplia en la quinta sección; la sexta, analiza los resultados obtenidos en los diferentes modelos que se aplicaron para poner a prueba la hipótesis de regresividad. Finalmente, se ofrecen algunas conclusiones y sugerencias para una política catastral con el fin de que ésta pueda lograr las metas de todo régimen fiscal: eficacia, eficiencia y equidad.

\section{El marco contextual de la recaudación predial en México}

Uno de los temas que actualmente se encuentra en la agenda del Senado de la República en México es la reforma para la modernización de los catastros en México. Se perfila un rol más activo del gobierno federal en materia catastral, esto considerando que los gobiernos de los estados y municipios en México no tienen incentivos para mejorar la recaudación ya que reciben, por medio de los acuerdos de coordinación y vía los ramos presupuestales 28 y 33, la mayor parte de sus ingresos, lo que suma alrededor de 71,1\% (Banobras, 2013). Las transferencias a los municipios suman alrededor de 1,4\% del producto interno bruto (PIB) (ColmenaresPáramo, 2005).

A manera de contexto, el impuesto predial que se recauda en México es equivalente a $0,3 \%$ del PIB comparado con $1,8 \%$ que los países miembros de la Organización para la Cooperación y el Desarrollo Económico (OCDE) recaudan, la Unión Europea y América del Norte 2\%, y América Latina $0,5 \%$ (Banobras, 2013). Además, se estima que el rendimiento del impuesto predial por predio en el país es de $\$ 649.78$ (Santana-Loza, 2006: 213).

El impuesto predial se considera un impuesto tipo ad valorem y se determina a partir del valor de la propiedad; es calculado por la oficina de catastro y, normalmente, los gobiernos locales son quienes tienen la atribución de administrar los catastros dado que son los que mejor conocen la dinámica del mercado de bienes raíces o inmuebles.

En una encuesta del Instituto para el Desarrollo Técnico de las Haciendas Públicas (Indetec) se encontró que en 572 municipios (24\%) la función de administración del catastro es realizada por el estado; 590 municipios (24\%) lo administran ellos mismos y 1015 , que son $42 \%$, 
está en manos de institutos catastrales; en 252 casos, que representan 10\%, la función es realizada en forma mixta (Santana-Loza, 2006: 214). En términos generales, de los impuestos propios que los gobiernos locales recaudan, que equivalen aproximadamente a 30\% del total, el impuesto predial representa 66,2\% (Banobras, 2013).

Según los datos de la Secretaría de Hacienda y Crédito Público, en 2011 solamente cuatro entidades federativas recaudaron 55\% del monto por concepto de impuesto predial: el Distrito Federal, Quintana Roo, Baja California Sur y Querétaro. El promedio per cápita de recaudación por concepto del predial fue de 205 pesos. La capacidad de recaudación del estado de Chihuahua, donde se localiza Ciudad Juárez, es superior al promedio nacional, ya que se recaudan aproximadamente 300 pesos per cápita.

El Banco Nacional de Obras y Servicios Públicos en el contexto del Plan Nacional de Desarrollo 2013-2018 y la meta nacional de crear un "México Próspero" se ha dado a la tarea de implementar una política de modernización catastral a través de convenios de acuerdo. Actualmente se han incorporado a dicho esfuerzo 93 municipios de nueve estados. Según datos del mismo Banobras, 25 municipios han concluido con sus esfuerzos de modernización y el programa se considera exitoso dado que ha logrado incrementar 50\% la capacidad recaudatoria. Los municipios exitosos donde la recaudación se ha incrementado sustantivamente son Colima (86\%), Manzanillo (78\%), Othon P. Blanco (76\%) y Carmen (76\%). El costo-beneficio se calcula superior a nueve pesos en los municipios más exitosos; el costo-beneficio significa que por cada peso invertido en la modernización se recaudaron nueve. Uno de los casos más exitosos de modernización catastral es el Distrito Federal, donde se asienta la capital nacional. Aquí la modernización se ha llevado a cabo por medio de la fotogramétrica para mejorar la información y tasas más progresivas en los segmentos más altos de vivienda; la tasa porcentual anual de crecimiento del impuesto predial aumentó de 5,5\% de 1991 al 2000 a $7,5 \%$ de 2000 a 2008.

No hay duda que la modernización catastral con fines recaudatorios es necesaria; sin embargo, no es suficiente, ésta debe ir acompañada de un análisis y evaluación de los efectos de equidad; es decir, no solamente debe tener una meta de efectividad y eficiencia recaudatoria, sino también de equidad en el impuesto. En la siguiente sección se discute el tema de equidad. 


\section{La cuestión de equidad en la política fiscal y el impuesto predial}

En términos generales, la equidad de cualquier política fiscal se juzga a partir de dos estándares: vertical y horizontal (Holcombe, 1983). Desde la perspectiva de un impuesto predial, lo importante es determinar el grado de progresividad o regresividad del impuesto mismo. Existe una opinión en común entre los expertos: todas las propiedades inmuebles deben ser valuadas de igual manera y pagar un impuesto proporcional acorde a su valor de mercado. La justificación de tener una estructura progresiva del impuesto predial es que aquellas propiedades que más valen consumen una mayor cantidad de los servicios públicos (alumbrado, pavimentación, policía, drenaje) que el gobierno oferta, consecuentemente deben pagar, vía el impuesto predial, por los mismos (Levy, 1995; Slack, 2013). Además, la inversión pública genera una plusvalía en la propiedad que el gobierno necesita captar por medio del impuesto predial (Smolka y Furtado, 2001).

Es importante diferenciar entre las formas de equidad vertical y horizontal (Holcombe, 1983); en la primera, desde la perspectiva del impuesto predial, existe de manera sistemática una variación entre los valores estimados y los valores de mercado de manera transversal en un rango de valores; es decir, puede darse el caso que propiedades de rangos bajos de manera consistente son valuadas a un precio superior del mercado comparado con propiedades con valores altos que serían subvaloradas (Kochin y Parks, 1982; Allen, 2003). Cuando estos errores ocurren de manera consistente se concluye que el impuesto predial es regresivo: paga más el que menos tiene. Lo opuesto es verdad si las propiedades en el rango más alto son valoradas consistentemente a un precio superior al del mercado comparativamente con las propiedades de rangos bajos; entonces, lo que se da es un impuesto progresivo.

La inequidad horizontal se define como aquella variación sistemática entre el valor estimado y el precio de mercado de aquellas propiedades que son similares en su valor de mercado; la fuente del error es el propio método de valuación inmobiliaria, que no es capaz de tomar en cuenta los impactos de ciertas propiedades o factores relacionados con características del vecindario (Allen y Dare, 2002). Allen (2003) también lo define como un impuesto base diferente entre propiedades con un valor de mercado similar.

En términos de las metas o los estándares sobre los cuales se juzga la equidad del impuesto predial en una jurisdicción se señala: el sistema impositivo se considera equitativo siempre y cuando todas las propiedades en la jurisdicción son gravadas en la misma proporción a su valor de 
mercado; es decir, todas las propiedades, independientemente de su valor o tipo, deben ser gravadas con la misma tasa efectiva (Allen y Dare, 2002).

\section{La equidad y el impuesto predial: aspectos estadísticos y econométricos}

Los estudios que han analizado y estimado la inequidad del impuesto predial normalmente se fundamentan en análisis de índole estadístico y métodos econométricos de cuadrados mínimos ordinarios. A continuación se explican los más comunes, que son citados por los expertos en el tema.

La propuesta de la Asociación Internacional de Valuadores Inmobiliarios, también conocida como IAAO por sus siglas en inglés, es una forma tradicional de estimar la equidad en el impuesto predial. Esta propuesta se basa en el análisis de los patrones que exhiben los datos del cociente de valuación (CV) que toma en cuenta los valores catastrales de la propiedad $\mathrm{i}\left(\mathrm{AV}_{\mathrm{i}}\right)$ con respecto a los valores de la transacción de mercado de la propiedad i $\left(\$ \mathrm{~V}_{\mathrm{i}}\right)$.

$$
\mathrm{CV}_{\mathrm{i}}=\frac{\mathrm{AVi}}{\$ \mathrm{Vi}}
$$

La equidad horizontal de esta medida se lleva a cabo analizando la dispersión de los datos del cociente conocido como el coeficiente dispersión. La equidad vertical mide la diferencia CV entre los valores altos y bajos de la muestra o población (Parker et al., 2011).

Por su parte Allen y Dare (2002) proponen que la equidad horizontal se mida a partir de la diferencia del valor absoluto del cociente de valuación de la propiedad $\mathrm{i}\left(\mathrm{CV}_{\mathrm{i}}\right)$ con respecto a la media aritmética de la zona o unidad espacial de referencia (CV). Aquí se compara la valuación de cada propiedad con respecto a la media de la unidad de análisis.

$$
\begin{aligned}
& \text { INEQUIDADi }=\mid \mathrm{CVi}-\overline{\mathrm{CV}} \\
& \mathrm{CVi}=\frac{\mathrm{Ai}}{\mathrm{Si}} \text { y } \overline{\mathrm{CV}}=\sum \mathrm{CVi} / \mathrm{n}
\end{aligned}
$$

$\mathrm{Si} \mathrm{CVi} \neq \overline{\mathrm{CV}}>0$ entonces existe inequidad horizontal

Otro índice es el desarrollado por Sunderman et al. (1990) que se denomina Porcentaje de Equidad Vertical (PEV) y aquí la variación total es medida por la suma total de los cuadrados de los cocientes de valuación de todas las propiedades, tomando como referencia su media aritmética. El componente vertical se mide por la suma de los cuadrados de la diferencia de los cocientes de valuación entre los casos por arriba y debajo de la mediana. 


$$
\mathrm{PEV}=\sum(C V i-\overline{C V})^{2}
$$

Métodos de cuadrados mínimos ordinarios para calcular inequidad

El método de Paglin y Fogarty (1972) es considerado como nobel para evaluar la equidad vertical en el mercado inmobiliario. El intercepto en la ecuación es el principal indicador para determinar inequidad; el intercepto debe ser estadísticamente significativo diferente a 0 (ecuación 5). Se utilizan el valor evaluado (AV) y el valor de venta (\$V) en el modelo. Aquí, si el intercepto es negativo y significativo indica una estructura progresiva, mientras que uno positivo y significativo estadísticamente capta una estructura regresiva (Allen, 2003).

$$
\mathrm{AV}=\mathrm{a}_{0}+\mathrm{a}_{1} \$ \mathrm{~V}
$$

Kochin y Parks (1982) proponen un modelo logarítmico con el valor de mercado como la variable explicativa y el valor estimado o catastral como dependiente. El argumento es que la relación no es lineal sino tiene una forma logarítmica. Entonces, al transformar las variables en logaritmos naturales se reduce el error y es factible entonces usar una regresión lineal.

$$
\operatorname{Ln} \$ V=a_{0}+a 1 \operatorname{Ln} A V
$$

Cheng (1974) utiliza el mismo modelo de Kochin y Parks (1982) de logaritmos naturales (ecuación 6). La diferencia es que el autor invierte las variables dependiente e independiente argumentando que el valor de venta es un mejor pronóstico del valor catastral (VA). En el modelo se utiliza el coeficiente de la pendiente para determinar si la estructura del impuesto es regresivo (el coeficiente es menor a 1) o progresivo (el coeficiente es mayor a 1).

$$
\operatorname{Ln} A V=a 0+a 1 \operatorname{Ln} \$ V
$$

El modelo de Bell (1984) a diferencia de Cheng agrega un término cuadrático en el valor del mercado para tomar en consideración la no linealidad de la función (citado en Sirmans et al. 1995). En otras palabras el valor cuadrático nos mide la velocidad del cambio; si los valores aumentan o disminuyen a una tasa creciente o decreciente.

$$
A V=a_{0}+a_{1} \$ V+a_{2} \$ V^{2}
$$


La IAAO (1996) propone un método en el cual la variable dependiente es el cociente de valuación y el precio del mercado como la variable dependiente. Si el coeficiente es 0 y no significativo indica que no existe inequidad. Aquí, si el valor del coeficiente $\mathrm{a}_{1}$ es mayor a 0 existe regresividad; pero si el valor es menor a 0 existe progresividad.

$$
\mathrm{AV} / \$ \mathrm{~V}=\mathrm{a}_{0}+\mathrm{a}_{1} \$ \mathrm{P}
$$

El método desarrollado por Clapp (1990) está basado en una técnica de cuadrados mínimos ordinarios en dos etapas (two stage). En la primera etapa se utiliza una variable instrumental, Z, que está altamente correlacionada con el valor estimado o de mercado, pero no con los errores del modelo. La variable $\mathrm{Z}$ se calcula clasificando los valores evaluados o catastrales (AV) en tres grupos iguales; a los valores del tercio más bajos se les asigna -1 mientras que al tercio más alto 1 y aquellos valores intermedios 0 . En la primera etapa la variable instrumental $Z$ se convierte en la variable independiente y el logaritmo natural del AV es la variable dependiente. La segunda etapa consiste en usar los valores pronosticados de la primera etapa como variable independiente en el valor de mercado como dependiente, como se presenta en las ecuaciones $10 \mathrm{y}$ 11. Para determinar la inequidad se utiliza el coeficiente $\mathrm{a}_{1}$; si el coeficiente es mayor a 1 y es estadísticamente significativo indica que el sistema es regresivo. Mientras que un valor menor a 1 y estadísticamente significativo significa una estructura progresiva.

$$
\begin{gathered}
\operatorname{Ln} A V=b_{0}+b_{1} Z \\
\operatorname{Ln} \$ V=a_{0}+a_{1} \operatorname{Ln} A V
\end{gathered}
$$

Sunderman y sus coautores (1990) proponen un modelo que incorpora una estructura diferenciada del mercado inmobiliario. La novedoso del método es que el mercado inmobiliario lo segmenta en tres grupos (alto, medio y bajo) con variables dicotómicas ( 1 y 0 ). Posteriormente se agrega una variable como un factor interactivo para evaluar si el intercepto de los valores altos y bajos son diferentes al valor medio o grupo de referencia (ecuación 12).

$$
A V=a_{00}+a_{10} \$ V+a_{01} L+a_{02} H+a_{11} L S+a_{12} H S
$$

Donde:

$\mathrm{L}=$ valores bajos

$\mathrm{H}=$ valores altos 
LS (valor de venta del grupo bajo, cero si no pertenece) LH (valor de venta del grupo alto, cero si no pertenece)

La interpretación de los coeficientes es más compleja; la prueba de hipótesis de no regresividad vertical es que $\mathrm{a}_{00}=\mathrm{a}_{01}=\mathrm{a}_{02}=0$. Sin embargo, si $\mathrm{a}_{00}>0$, el impuesto es regresivo en el rango medio del mercado. Si $\mathrm{a}_{00}<0$ el impuesto es progresivo en el rango medio. Los coeficientes para $\mathrm{H}$ y L solamente nos dicen si el intercepto de los rangos alto y bajo son diferentes al rango de referencia o medio (Sirmans et al, 1995).

\section{Datos y aspectos metodológicos}

Ciudad Juárez, en las últimas tres décadas (1980-2010), experimentó un proceso de desarrollo urbano acelerado a raíz de la inversión extranjera directa (IED) en la forma de maquiladora o plantas ensambladoras de capital casi cien por ciento extranjero. Esta IED generó empleo y con ello altas tasas de migración, reflejándose en el crecimiento de la población y la mancha urbana; la población creció, en términos absolutos, de 567,000 habitantes a 1,300,000 de 1980 a 2005; la mancha urbana de 9,300 a 31,246 hectáreas (Fuentes-Flores, 2001).

El crecimiento de la mancha urbana ha generado la necesidad de mejorar no solamente los procesos de gestión del desarrollo urbano, sino también la de modernizar el sistema catastral para mantener actualizados los valores del suelo y, de esa manera, captar recursos que permitan financiar la inversión en equipamiento e infraestructura (Рeña et al. 2012).

La mayor parte de los estudios que analizan la equidad del impuesto predial se basan en datos individuales de las propiedades y toman en consideración el valor que el catastro asigna a la propiedad y el valor de venta de la misma; es decir, se espera contar con ambos datos de la propiedad. La accesibilidad de ambos datos no es problemático en países desarrollados tales como Estados Unidos (Sirmans et al., 1995). Sin embargo, la accesibilidad de datos en países en vías de desarrollo es más problemático dado que la información de ventas es escasa o inaccesible. La información sobre las transacciones que los catastros tienen, si existe, están subvaluadas ya que los compradores y vendedores tienen el incentivo de dar información incorrecta que les favorezca para propósitos gravables.

Este estudio se basa en dos fuentes de información: una es la oficina local de catastro y datos del valor de las propiedades en la que se basan para determinar el impuesto predial. Se consideraron 427 predios para este estudio. Esta fuente de información se utilizó para generar los datos de valuación o estimados (AV) que en los diferentes modelos emplean; la 
otra fuente en venta en los anuncios clasificados es el periódico local $E l$ Diario y los sitios de internet de las diferentes empresas de bienes raíces donde se integren.

Los datos sobre el valor de mercado ( $\$ \mathrm{~V}$ en los modelos) se basan en información del 2011; se recolectaron datos de 427 propiedades. ${ }^{1}$

Es importante clarificar que los datos de propiedades de catastro (AV) y los anuncios de venta o mercado ( $\$ \mathrm{~V})$ no son los mismos; esto plantea el problema de cómo comparar dos datos disimiles. Los sistemas de información geográfica (SIG) y análisis espacial son una herramienta importante para resolver el problema de contar con fuentes de información diferente. Kriging Ordinario (KO) es una herramienta de análisis espacial, del paquete ArcMap 9.3, que permite resolver el problema de los datos. $\mathrm{El} \mathrm{KO}$ permite transformar información cartográfica de puntos $(\mathrm{x}, \mathrm{y}) \mathrm{a}$ polígonos de superficie. El KO se basa una técnica de interpolación para hacer pronósticos de valores de datos que no se conocen con base en otros datos, utilizando el concepto de vecinos cercanos y la distancia inversa ponderada (Smith de et al. 2007). Un principio básico de la correlación espacial, basada en la ley de Tobler, es que todo está correlacionado; sin embargo, existe mayor similitud entre las cosas más cercanas; esto significa que el mejor pronóstico del valor de una propiedad es aquél de la propiedad vecina. El método de Krigin Ordinario (KO) se basa en este principio espacial para el pronóstico de los valores de la propiedad, además, es apropiado para datos de tendencias que estiman parámetros en un punto de tiempo, mientras que el Krigin Universal lo es más para datos de superficie dinámica y estudio de tendencias.

El proceso metodológico se explica de mejor manera en el diagrama que a continuación se presenta (figura 1). El proceso inicia con datos de diferentes fuentes en la forma de puntos de localización de las viviendas basado en ordenadas y coordenadas (x,y). El siguiente paso es aplicar la técnica de $\mathrm{KO}$ para transformar los datos (catastrales y venta) de puntos $(\mathrm{x}, \mathrm{y})$ a polígonos, tomando como base el mapa de unidades espaciales de los distritos que maneja la oficina de catastro de Ciudad Juárez. Los datos de catastro $(\mathrm{AV})$ y los datos de los anuncios de precios de venta $(\$ \mathrm{~V})$ se unen utilizando la unidad espacial de los distritos de catastro. Finalmente, se procede al análisis estadístico y econométrico. En resumen, la unidad de análisis de equidad no es el predio individuales sino las unidades espaciales o distritos de catastro $(\mathrm{N}=299)$. Los datos AV son el valor pronosticado o promedio de la unidad espacial de cada distrito o unidad catastral. El análisis parte del supuesto que los distritos son unidades es-

${ }^{1}$ Para más detalle consultar a Peña et al, 2012. 
paciales homogéneas; en términos generales los datos se comportan de tal manera por lo que el análisis tiene validez.

\section{Figura 1}

\section{Proceso metodológico}
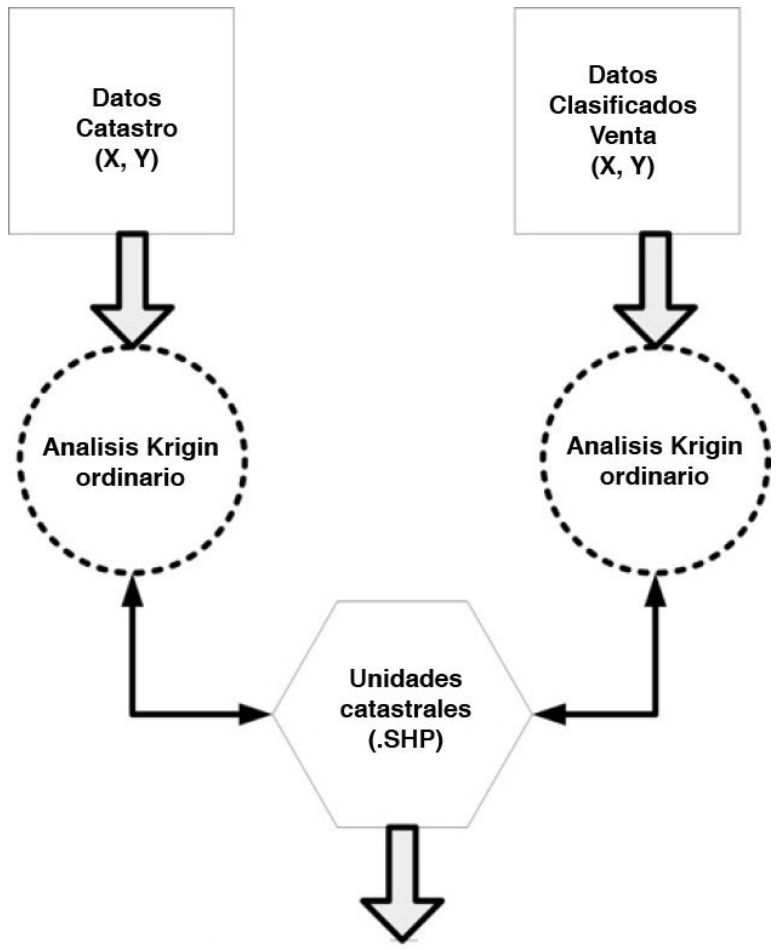

\section{Resultados estimados por} unidades catastrales

Fuente: elaboración propia.

\section{Resultados}

La figura 2 muestra los resultados obtenidos una vez que se calcularon ambos valores: catastral (AV) y precio de venta o mercado (\$V). Los valores en el mapa son el porcentaje que representa el valor catastral con respecto al precio de venta. Los datos más claros representan una subvaluación mientras que los datos más oscuros son datos sobrevaluados por el catastro. Mientras más cercano a uno el valor indica que el valor catastral y el precio de venta se aproximan (colores grises). Cada polígono en 
el mapa representa una unidad espacial que la oficina de catastro usa para propósitos propios.

\section{Figura 2}

Valores unidades catastrales

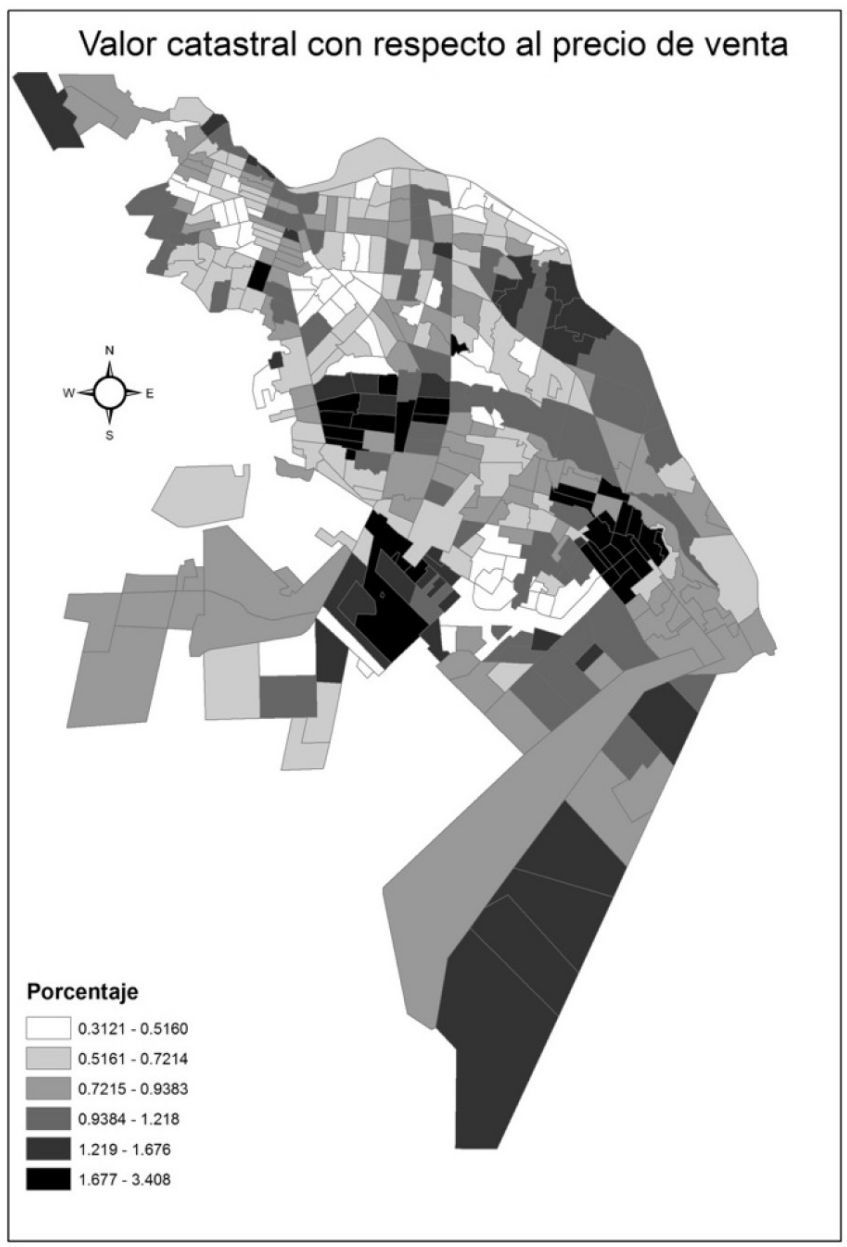

Fuente: elaboración propia.

Es importante señalar que los datos subvaluados se localizan en su mayoría en la parte norponiente de la ciudad, mientras que los más sobrevalorados se localizan agrupados en diferentes zonas de la ciudad. La explicación es que la zona norponiente se desarrolló en sus orígenes con asentamientos irregulares, pero con el paso de los años han existido mejoras a un ritmo más acelerado que la capacidad de los sistemas catastrales para actualizar sus valores. Los sobrevalorados no se pueden entender 
sin tomar en cuenta que el año de los datos de precios de venta es 2011, justo cuando la ciudad enfrenta un fenómeno de violencia sin precedentes a causa de la lucha de los carteles de la droga por controlar el flujo en esa zona.

Esas zonas pueden considerarse estables, con habitantes de clase media y alta que, en el contexto de la violencia, emigraron a la ciudad vecina en El Paso, Texas en Estados Unidos o salieron de la ciudad al interior de México ofertando las viviendas a un precio muy por debajo del mercado en condiciones normales. Los catastros siguen evaluando las propiedades sin tomar en cuenta estos factores, lo que implica una consecuente sobrevaloración.

En el cuadro 1 se muestran las estadísticas descriptivas de ambas variables de interés que son el valor catastral $(\mathrm{AV})$ y el precio de venta $(\$ \mathrm{~V})$ de las unidades espaciales catastrales. Uno de los aspectos importantes a destacar es que el rango de valores es mayor en los datos de precios de venta en comparación con los valores catastrales. Segundo, la media aritmética de los valores de precio de venta es superior al del valor catastral; los valores catastrales son inferiores en 20 por ciento. Esta diferencia no es inusual, ya que los valores catastrales son estimadores del precio de mercado por lo que siempre existirá un mayor error. En total existen 379 unidades espaciales; sin embargo, para propósito de análisis excluimos los extremos por las razones anteriormente expuestas de la sub y sobrevaloración, se enfocan entonces las unidades (299) más estables en términos de sus valores. Este procedimiento aumentó el valor del coeficiente de determinación de $10 \%$ a aproximadamente $50 \%$ (cuadro 2 ).

\section{Cuadro 1 \\ Estadísticos descriptivos}

\begin{tabular}{lccccc}
\hline & $N$ & Minimo & Máximo & Media & D. Estándar \\
\hline AV (valor catastro) & 299 & $292,031,49$ & $1,519,797,49$ & $519,567,56$ & 225804,35 \\
\$V (valor venta) & 299 & $248,354,58$ & $1,90,5758,85$ & $658,112,67$ & 260529,18 \\
N válido (según lista) & 299 & & & & \\
\hline
\end{tabular}

Fuente: elaboración propia.

El cuadro 2 muestra los resultados de los diferentes modelos que se discutieron previamente y que se consideran como los más avanzados para poner a prueba la hipótesis de regresividad en el sistema del impuesto predial. A continuación se discuten los aspectos más importantes a destacar a partir de los resultados de los modelos. 


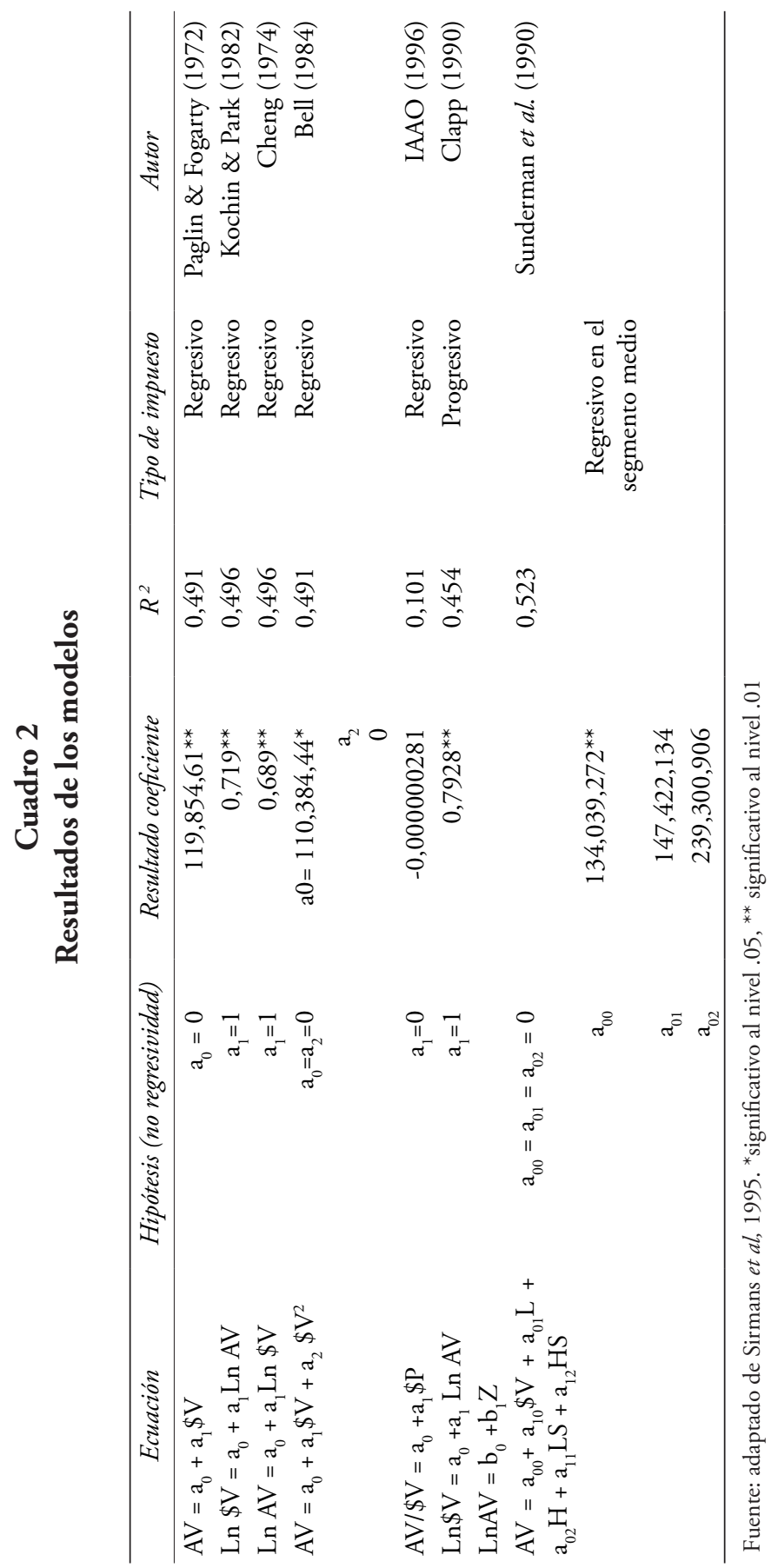


El primer aspecto a destacar y discutir es el coeficiente de determinación o mejor conocido como $\mathrm{R}$ cuadrada. Estudios en países avanzados que han replicado los mismos modelos en otros lugares encuentran $\mathrm{R}$ cuadradas cercanas a 1 que significa que los modelos tienen un mínimo de error. Por ejemplo, Sirmans y coautores (1995) en el contexto de Miami, Florida encuentra R cuadradas superiores a 90 por ciento; Allen (2003) en el caso de Fort Lauderdale, Florida encuentra R cuadradas que van de un mínimo de $4,4 \%$ para el modelo IAAO a un máximo de $90 \%$ para el modelo de Cheng. Los resultados para Ciudad Juárez encuentran consistencia, dado que el modelo IAAO tiene una $\mathrm{R}$ cuadrada de $10 \%$ que es la más baja y $52 \%$ para el caso del modelo de Sunderman et al. (1990); el coeficiente de determinación es consistente alrededor del 50 por ciento.

La diferencia en el coeficiente de determinación entre los casos de países avanzados y el que aquí se estudia se explica en gran medida por la calidad de los sistemas catastrales; los valores de catastros en Estados Unidos en términos generales son una buena proxy de los precios de venta o mercado, en gran parte esa eficiencia se debe a que la información es accesible y los catastros son más profesionales y efectivos. En el caso de Ciudad Juárez los valores catastrales y los valores de precios de venta o mercado presentan un mayor error, lo que significa que se necesita un mayor esfuerzo para mejorar la valuación inmobiliaria y del suelo por medio de la modernización catastral y los métodos de valuación.

Un segundo aspecto a destacar es el resultado con respecto a la hipótesis de regresividad en el sistema predial. De manera consistente, con excepción del modelo de Clapp, las pruebas de hipótesis indican que en Ciudad Juárez la estructura del impuesto predial es de tipo regresivo. Es decir, la estructura es inequitativa dado que los segmentos altos reciben un mejor trato que los segmentos bajos en términos proporcionales o relativos. Con base en los resultados se rechaza la hipótesis de trabajo de no regresividad por la hipótesis alternativa de regresividad. El modelo de Sunderman et al (1990) es aún más ilustrativo, dado que indica que los segmentos medios ${ }^{2}$ son los que mayor carga impositiva pagan comparado con los segmentos bajos y altos.

Un tercer aspecto importante a destacar es una debilidad en los modelos, ya que miden la regresividad a partir de la comparación entre los valores de catastro y los valores de ventas. Si existe una diferencia significativa, entonces se asume que los impuestos no son los adecuados o están sujetos a errores, además, se parte del supuesto que los valores de

\footnotetext{
${ }^{2}$ En el modelo los segmentos bajo, medio y alto se calcularon utilizando los quiebres naturales de Jenks que son parte de la paquetería ArcMap. Esta herramienta analítica permite identificar clúster de datos y ubicar donde se dan los cortes dependiendo los grupos que se quieran.
} 


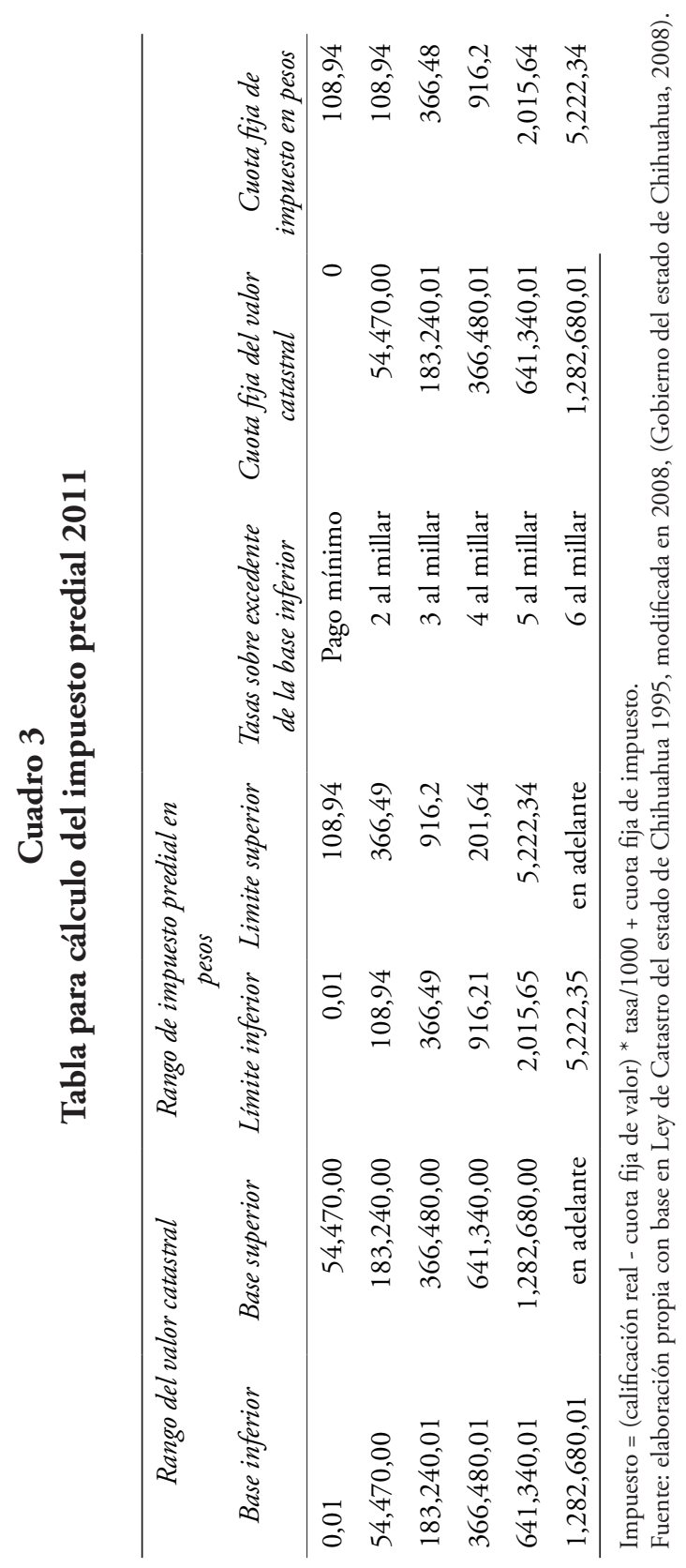


catastro determinan el nivel de impuesto. Sin embargo, lo ideal sería saber el valor del pago del impuesto y contrastarlo, ya sea con el valor de catastro o de venta, para ver si existe regresividad. Un sistema progresivo sería aquél cuya tasa es mayor en aquellos segmentos cuya propiedad es más valorada.

A continuación se pone a prueba otro modelo para determinar la regresividad a partir del cálculo del impuesto predial utilizando la fórmula que el municipio de Juárez emplea para determinar el impuesto predial (cuadro 3). Para cada unidad de análisis catastral se calculó el impuesto potencial aplicando la fórmula del cuadro 3. Esto ofrece una proxy del impuesto y una estimación de la tasa del mismo.

El modelo es el siguiente:

$\operatorname{Ln} T=a_{0}+a_{1} \operatorname{Ln} A V+a_{2} \operatorname{Ln} A V^{2}$

Donde

$\mathrm{T}=$ impuesto

$\mathrm{AV}=$ valor catastral

$\mathrm{AV}^{2}=$ valor catastral al cuadrado

$\mathrm{Ln}=$ logaritmo natural

La hipótesis que se pone a prueba de no regresividad es que $\mathrm{a}_{2}>0$. El coeficiente $a_{1}$ simplemente indica la tasa efectiva de impuesto, mientras que el coeficiente $\mathrm{a}_{2}$ mide si la tasa aumenta conforme el valor catastral se incrementa. Se utilizan ambos logaritmos para interpretar los coeficientes como la elasticidad o el cambio porcentual (cuadro 4).

\section{Cuadro 4 \\ Resultados del modelo 13}

\begin{tabular}{|c|c|c|c|c|c|c|}
\hline & \multirow[t]{2}{*}{ Modelo } & \multicolumn{2}{|c|}{ Coeficientes no estandarizados } & \multirow{2}{*}{$\begin{array}{c}\text { Coeficientes } \\
\text { estandarizados } \\
\text { Beta }\end{array}$} & \multirow[t]{2}{*}{$t$} & \multirow[t]{2}{*}{ Sig. } \\
\hline & & $B$ & std. error & & & \\
\hline & (Constant) & $-15,402$ & 0,569 & & $-27,048$ & 0,000 \\
\hline \multirow[t]{2}{*}{1} & $\ln A V$ & 2,046 & 0,086 & 1,458 & 23,824 & 0,000 \\
\hline & $\ln A V^{2}$ & $-0,024$ & 0,003 & $-0,458$ & $-7,488$ & 0,000 \\
\hline
\end{tabular}

a. Dependent Variable: LnIMPUESTO R cuadrado = 1

Fuente: elaboración propia con base en los datos de las tablas de valores de la Ley de Catastro (ver figura 3). 
La figura 3 presenta en términos porcentuales el impuesto y cómo se distribuye en la ciudad. Esto nos dará un mejor panorama de la regresividad y su distribución espacial. Los datos dividen la ciudad en cuatro grupos o cuartiles; los datos son la proporción del impuesto que se paga con respecto al valor catastral de la zona. La parte poniente, -colores claros-, es la que menos paga y el norponiente, -colores obscuros-, la que más paga, proporcionalmente hablando con respecto al valor catastral.

\section{Figura 3}

\section{Proporción del impuesto predial respecto al valor catastral}

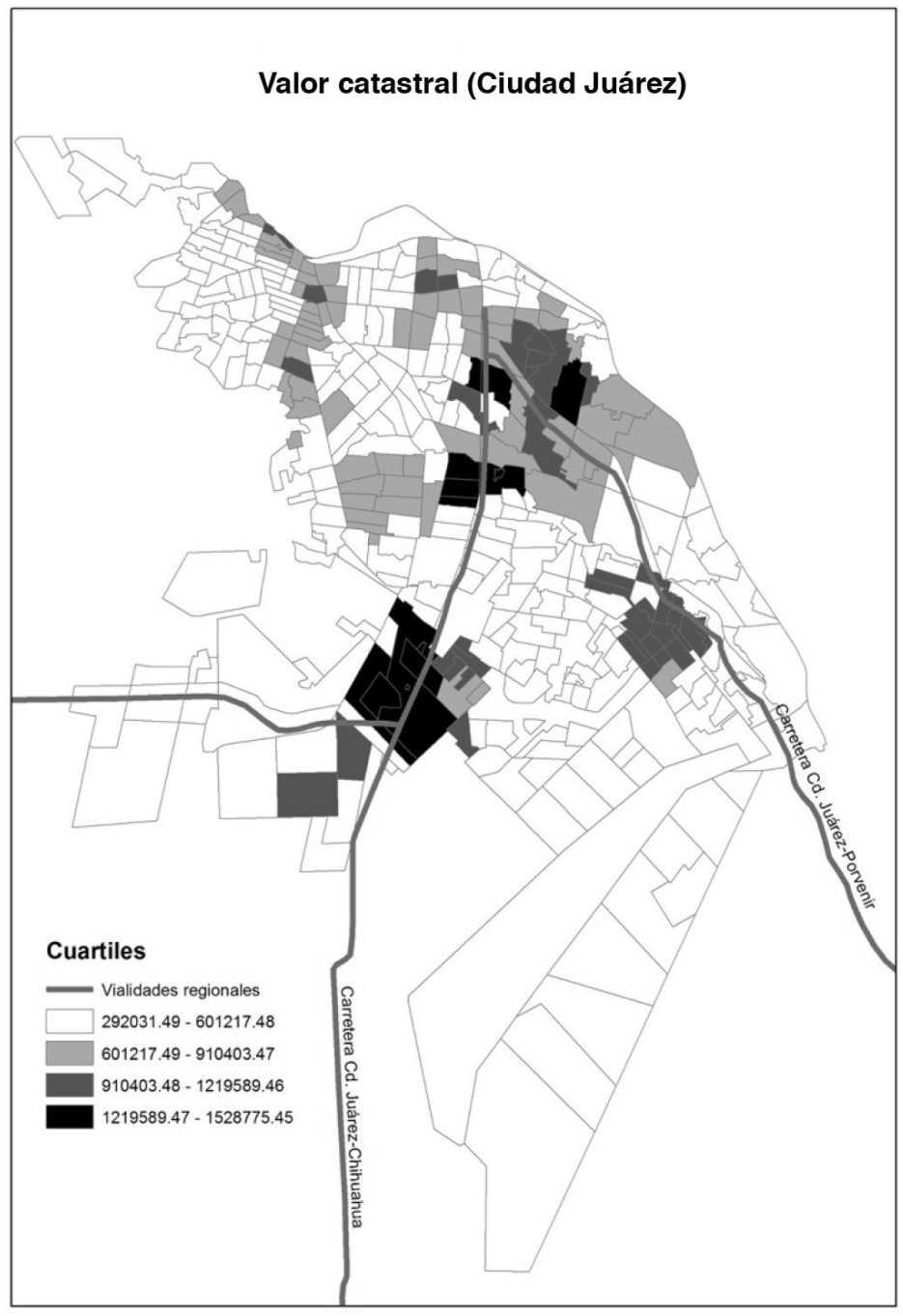

Fuente: elaboración propia. 
Los resultados del modelo indican (cuadro 4) una progresividad según la hipótesis de Paglin y Fogarty (1972), ya que el intercepto no es 0 y estadísticamente significativo. Las zonas cuyos valores son inferiores en un cierto rango están exceptuadas del impuesto. Sin embargo, a partir de cierto rango es importante saber si las zonas cuyo valor catastral es más alto pagan un impuesto progresivo. El modelo no puede rechazar la hipótesis de regresividad. El parámetro $\mathrm{a}_{2}$ es negativo (-.024) y significativo. Esto indica una tasa decreciente conforme los valores catastrales aumentan; es decir, si el valor catastral aumenta $1 \%$ la tasa marginal del impuesto disminuye en 0,024 por ciento.

\section{Conclusiones y propuestas de política pública}

Este artículo es un esfuerzo pionero en México para poder analizar aspectos de equidad en el impuesto predial. Existen trabajos que hablan sobre la necesidad de desarrollar esfuerzos a nivel municipal para la captura de la plusvalía (Smolka y Furtado, 2001) o mejorar los sistemas de valoración a partir de herramientas de técnicas de valuación masiva (Perló-Cohen y Zamorano-Ruiz, 1999), o bien, valuación inmobiliaria por medio de modelos hedónicos (Fitch-Osuna, 2010); sin embargo, no tengo conocimiento de trabajo alguno que de manera sistemática y cuantitativa analice aspectos de equidad como aquí se hace.

El trabajo logró el objetivo de presentar evidencia de la regresividad de la política fiscal del impuesto predial. Los sistemas SIG ofrecen una herramienta importante para llevar a cabo análisis espacial en corto tiempo y con pocos recursos, haciendo factible evaluar la estructura del impuesto de propiedad no sólo en cuanto a la eficiencia y efectividad sino también de equidad.

El artículo y el tema son relevantes, particularmente cuando a nivel de la clase política el tema de los catastros municipales está siendo discutido en el Senado de la República. Se perfila que en el futuro el gobierno federal se asigne mayores atribuciones para la administración de los catastros municipales ante la incapacidad y falta de voluntad política de los municipios para modernizar sus catastros. Sin embargo, dicha reforma tienen un carácter más de índole recaudatorio que una visión de desarrollo urbano territorial. El Lincoln Institute of Land Policy (LILP) es un ejemplo de centro que ha promovido la idea de que el desarrollo urbano no está disociado del tema de regulación del suelo y el gravamen de la propiedad. El LILP, a través de sus seminarios permanentes, ha tenido presencia e influencia en Latinoamérica en general y México en particular.

Las ciudades en México requieren de un proyecto de planeación que revierta la especulación del suelo urbano y la segregación espacial, que son 
muy claras y marcadas en nuestro caso de estudio en Ciudad Juárez. La especulación del suelo produce dos resultados socialmente inaceptables: 1) la inacción de los municipios para gravar el suelo improductivo reduce las oportunidades económicas que pueden generar en la ciudad. 2) Los municipios no maximizan sus ingresos, ya que el pago del impuesto es menor al que potencialmente pudieran captar; al no maximizar los ingresos producto del suelo urbano no tienen suficientes recursos para financiar infraestructura y equipamiento urbano, y al no tener ingresos no es posible mejorar la accesibilidad de la población de bajos ingresos a los espacios lúdicos, oportunidades de empleo, entre otros aspectos de la población de más bajos recursos.

En resumen, es necesaria una política de impuesto predial que no solamente sea recaudatoria sino de tipo progresivo, en la cual las tasas marginales se incrementen en los estratos donde el suelo es más cotizado y donde viven los segmentos socioeconómicos más altos. Un caso donde este tipo de política se ha dado es en la Ciudad de México, en el gobierno de Marcelo Ebrard, donde se incrementaron las tasas marginales en las zonas más caras de la ciudad.

Ciudad Juárez se caracteriza por ser una ciudad en la que la segregación espacial es muy marcada y los segmentos de más bajos recursos tienen la peor accesibilidad. Además, existen lotes baldíos en número importante en los que la especulación es business as usual. Una política predial progresiva podría ayudar a revertir esta situación.

La política pública que se propone con relación al impuesto de la propiedad es la siguiente: 1 . se deben mejorar y profesionalizar los sistemas de valuación catastral, ya sea mediante un método masivo (Peña et al. 2012) o un método que no sea costoso y que requiera de un alto número de trabajadores/hora. 2. Es necesario tener las herramientas legales que le permitan a los municipios implementar valuaciones masivas. 3. Es importante que las evaluaciones de desempeño de los catastros no sólo se vean desde el punto de vista recaudatorio y de eficiencia, la equidad debe ser un elemento que debe también tomarse en consideración, tal como se ha hecho claro en este estudio. 4. Reflexionar sobre qué institución u órgano es el más adecuado para implementar una reforma catastral; es claro que los gobiernos locales o estatales no tienen el incentivo de usar la idea de mas impuestos como plataforma política y aunque el gobierno federal se ha anotado para hacerlo es importante pensar si es lo mas adecuado. Se debe explorar la alternativa de institutos descentralizados y autónomos que puedan llevar a cabo los procesos de valuación o darle mas peso a las asociaciones profesionales de valuadores inmobiliarios. 5 . Vincular la política fiscal de los impuestos de propiedad con una política 
de desarrollo urbano enfocada a la captura de plusvalía que pueda socializarse con el objetivo de dar mantenimiento a espacios públicos y mejorar la accesibilidad de las poblaciones vulnerables al derecho de la ciudad.

\section{Fuentes consultadas}

Allen, Marcus y William Dare (2002), "Identifying determinants of horizontal property tax inequity: evidence from Florida", Journal of Real Estate Research, 24 (2), American Real Estate Society, Clemson, pp. 153-164.

Allen, Marcus (2003), "Measuring vertical tax propert inequity in multifamily property markets", Journal of Real Estate Research, 25 (2), American Real Estate Society, Clemson, pp. 171-184.

Banobras (Banco Nacional de Obras y Servicios Públicos) (2013), "Programa de Modernización Catastral”, Banobras, México, <http:// www.banobras.gob.mx/productosyservicios/productos/asistenciatecnicayfinanciera/Paginas/ProgramadeModernizaci\%C3\%B 3nCatastral.aspx>, 25 de agosto de 2013.

Bell, Earl (1984), "Administrative inequity and property assessment: the case of traditional approach", Property Tax Journal, 3 (2), Lincoln Institute of Land Policy, Cambridge-Massachusetts, pp. 123-131.

Cheng, Pao Lun (1974), "Property taxation, assessment performance, and its measurement", Public Finance, 29 (3), Foundation Journal Public Finance, The Hague, Netherlands, pp. 268-84.

Clapp, John (1990), "A new test for equitable real estate tax assessment", Journal of Real Estate Finance and Economics, 3 (9), SpringerVerlag GmbH, Heidelberg, pp. 233-49.

Colmenares-Páramo, David (2005), "Equidad y responsabilidad fiscal, La experiencia mexicana”, Economía UNAM, 2 (4), Universidad Nacional Autónoma de México, México, pp. 40-69.

Fitch-Osuna, Jesús Manuel (2010), Emulación del valor inmobiliario y dinámica territorial, Universidad Autónoma de Nuevo León-UPC, Monterrey. 
Fuentes-Flores, César Mario (2001), "Los cambios en la estructura intraurbana de Ciudad Juárez, Chihuahua: de monocéntrico a multicéntrica”, Frontera Norte, 13 (25), El Colegio de la Frontera Norte, Tijuana, pp. 95-118.

Gobierno del Estado de Chihuahua (2008), "Reforma a la ley de catastro 1995", Gobierno del Estado de Chihuahua, Chihuahua.

Holcombe, Randall (1983), Public finance and the political process, Southern Illinois University Press, Carbondale.

IAAO (International Association of Assessing Officers) (1996), Property assessment valuation, IAAO, Chicago.

Kochin, Levis y Richard W. Parks (1982), "Vertical equity in real estate assessment: a fair appraisal”, Economic Inquiry, 20 (4), Wesley W. Wilson-University of Oregon, Eugene-Oregon, pp. 511-531.

Levy, John (1995), Essential microeconomics for public policy analysis, Praeger, Wesport-Connecticut.

Paglin, Morton y Michel Fogarty (1972), "Equity and the property tax: a new conceptual performance focus", National Tax Journal, 25 (4), National Tax Association, Houston, pp. 557-565.

Parker, David, Tony Lockwood y Wayne Marano (2011), "Mass appraisal certification standards-the spatial dimension", 17th Pacific rim real estate society conference, 16-19 January 2011, Gold Coast, Australia, pp. 1-17, <http://www.prres.net/papers/Lockwood_Mass_ Appraisal_Certification_Standards.pdf>, 13 de agosto de 2013.

Peña, Sergio, Cesar M. Fuentes, Luis Cervera y Vladimir Hernández (2012), "Planning support systems: a computer-assisted mass appraisal (CAMA) system for Ciudad Juárez, México”, Journal of Property Tax Assessment \& Administration, 9 (4), International Association of Assessing Officers and the International Property Tax Institute, Kansas, pp. 25-40.

Perló-Cohen, Manuel y Zamorano-Ruiz, Luis (1999), "Reform of the real estate tax system in Mexicali: 1989-1998”, working paper, Lincoln Institute of Land Policy. 
Santana-Loza, Salvador (2006), "El impuesto predial y el catastro en México", en Compilación de conferencias en seminarios internacionales indetec 2002-2005, Indetec, México, pp. 203-218.

Sirmans, Stacy, Barry Diskin y Swint Friday (1995), "Vertical inequity in the taxation of real property", National Tax Journal, 48 (1), National Tax Association, Houston, pp. 71-84.

Slack, Enid (2013), "The politics of the property Tax", en William J. McCluskey, Gary C. Cornia y Lawrence C. Walter (eds.), A primer on property tax: administration and policy, Wiley-Blackwell, Ames, pp. 69-88.

Smith de, Michel, Michel Goodchild, Paul Longley (2007), Geospatial analysis: a comprehensive guide to principles, techniques and software tools, Troubador Publishing Ltd, Leicester.

Smolka, Martim y Fernanda Furtado (2001), "Ensayo introductorio: recuperación de plusvalías en Latinoamérica: ¿̨bravura o bravata?”, en Recuperación de plusvalías en América Latina: alternativas para el desarrollo urbano, Eurelibros-Instituto de PosGrado e Investigación-Pontifícia Universidad Católica de Chile-Lincoln Institute, Santiago, pp. XIII-XLI.

Sunderman, Mark, John Birch, Roger Cannaday y Thomas Hamilton (1990), "Testing for vertical inequity in property tax systems", Journal of Real Estate Research, 5 (3), American Real Estate Society, Clemson, pp. 319-334.

Recibido: 7 de agosto de 2014. Corregido: 13 de enero de 2015. Aceptado: 21 de abril de 2015.

Sergio Peńa-Medina. Doctor en planificación urbana y regional por The Florida State University, Estados Unidos. Actualmente es profesor-investigador del Departamento de Estudios Urbanos y Medio Ambiente en El Colegio de la Frontera Norte en Ciudad Juárez, Chihuahua. Es miembro del Sistema Nacional de Investigadores, nivel II. Sus líneas de investigación actual son Teoría de la planeación, planificación transfronteriza y valuación catastral. Entre sus últimas publicaciones destacan, en coautoría: "Planning support systems: a Computer Assisted Mass Appraisal (CAMA) System 
for Ciudad Juarez, Mexico", Journal of Property Tax Assessment \& Administration, 9 (4), Association of Assessing Officers and the International Property Tax Institute, Kansas, pp. 25-40 (2013); "Recent trends and practice in spatial planning in Mexico: the municipal planning and research institutes", Gestión y Politica Pública, 21 (2), Centro de Investigación y Docencia Económicas, México, pp. 407-449 (2012), "Regímenes de planificación transfronteriza: México-Estados Unidos”, Región y Sociedad, XXIII (50), El Colegio de Sonora, Hermosillo, pp. 115-151 (2011). 